\title{
A chakana: dimensão histórica e interdisciplinaridade
}

Luiz Carlos de Andrade (D)

\author{
Rosa García Márquez (iD
}

Fábio Silva de Souza

\section{Resumo}

O presente trabalho é baseado em pesquisas bibliográficas e relatos de alguns arqueólogos e tem por objetivo apresentar uma breve resenha histórica sobre o símbolo andino conhecido como chakana, explorando alguns conceitos matemáticos através da análise dos aspectos geométricos deste símbolo, com a intenção de estimular os professores do Ensino Básico a criar e recriar sua prática de ensino e aprendizagem pautados nos princípios de interdisciplinaridade. É sugerido que o professor oriente os alunos em uma pesquisa sobre a história da chakana, a localização geográfica das antigas culturas que a utilizavam, além de analisar a Arte e Matemática dos povos andinos e principais culturas da América do Sul antes da colonização. Vestígios arqueológicos mostram a precisão matemática nas construções de templos, utensílios cerimoniais e principalmente a cosmovisão desses povos andinos. Com isso, esperamos agudizar a curiosidade do leitor, justificando as diversas formas de produção matemática nas antigas culturas, contribuindo assim, para um despertar dos nossos alunos, relacionado aos conceitos de Matemática do Ensino Básico de forma contextualizada com as descobertas de nossos ancestrais.

Palavras-chave: Geometria; Interdisciplinaridade; Teorema de Pitágoras; chakana.

\begin{abstract}
The present work is based on bibliographic research and reports by some archaeologists and aims to present a brief historical review of the Andean symbol known as chakana, exploring some mathematical concepts through the analysis of the geometric aspects of this symbol, with the intention of encouraging teachers Basic Education to create and recreate their teaching and learning practice based on the principles of interdisciplinarity. It is suggested that the teacher guide students in researching the history of chakana, the geographical location of the ancient cultures that used it, in addition to analyzing the Art and Mathematics of the Andean peoples and the main cultures of South America before colonization. Archaeological remains show the mathematical precision in the construction of temples, ceremonial utensils and especially the worldview of these Andean peoples. With this, we hope to sharpen the reader's curiosity, justifying the various forms of mathematical production in ancient cultures, thus contributing to an awakening of our students, related to the concepts of Mathematics in Basic Education in a contextualized way with the discoveries of our ancestors.
\end{abstract}

Keywords: Geometry; Interdisciplinarity; Pythagorean theorem; chakana. 


\section{Introdução}

Estamos em um mundo de formas. Para qualquer lugar que se direcione o olhar, as formas geométricas estão presentes, seja na natureza, nas artes, na arquitetura ou em outras áreas do conhecimento.

De acordo com a BNCC [3], "Reconhecer, comparar e nomear figuras planas (círculo, quadrado, retângulo e triângulo), por meio de características comuns, em desenhos apresentados em diferentes disposições ou em sólidos geométricos"é uma habilidade que os alunos devem desenvolver. Desta forma, temos os conceitos básicos da geometria como um dos conteúdos mais importantes para o Ensino Básico.

Segundo Andrade [1] explorar a matemática em símbolos de civilizações antigas apresentando sua história e mistérios e estudar os costumes desses povos, em especial os latino-americanos, pode aguçar a curiosidade dos alunos e estabelecer conexões da Matemática com outras áreas do conhecimento como Geografia, História, Ciências e Arte.

Conectar a geometria e a história das civilizações antigas fornece uma variedade de situações interdisciplinares a serem exploradas, tais como: pinturas artísticas (em tecidos e cerâmica), construções arquitetônicas de templos, terraças ${ }^{1}$ e a construção das cidades com pedras angulares de encaixes perfeitos que suportavam a ação do tempo e forças da natureza, como terremotos, ver Motta [7],.

Neste artigo abordamos um símbolo utilizado nas antigas civilizações andinas, conhecido pelo nome de chakana como ponto principal para a interdisciplinaridade.

\section{A Chakana}

Nossos ancestrais, eram movidos pela curiosidade observando os padrões na natureza, em especial os movimentos dos astros, ver Amaral [9]. Para esses povos tal observação era uma prática comum e não foi diferente para o povo andino: eles se inspiraram na constelação do Cruzeiro do Sul, composta pelas estrelas de Magalhães, Mimosa, Rubídea, Pálida e Intrometida, conforme pode ser visto na Figura 1, e criaram a chakana. Para Bazán, Villena e Ramos [2, 8, 10], ela é o símbolo base da cosmovisão andina.

\footnotetext{
${ }^{1}$ Técnica agrícola em terrenos muito íngremes, permitindo o seu cultivo e o controle da erosão.
} 


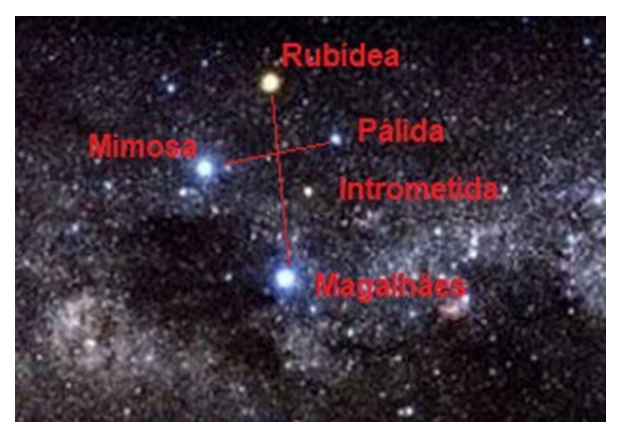

Figura 1: Constelação do Cruzeiro do Sul (adaptado)

Fonte:

http://prensalibrepueblosoriginarios.blogspot.com/2012/04/la-chakanaherencia-de-nuestros.html

Villena [8] menciona que entre o dia 2 e 3 de maio a constelação mencionada forma uma cruz perfeita, em posição vertical em relação ao Polo Sul. No dia 3 de maio, alguns povos andinos celebram o Dia da Chakana, agradecendo a colheita e cultivo de alimentos, [12].

A chakana "é uma figura geométrica usada há milhares de anos pelo povo andino que a nomeia. No fundo é uma forma geométrica que representa quatro escadarias de três degraus cada, com um círculo dividido ao meio na parte central do símbolo", [6].

De acordo com Ramos [10], como a chakana esteve presente em várias culturas andinas antigas antes da colonização europeia, ela assume algumas variantes em sua forma geométrica, sendo moldada e adaptada para cada uma dessas culturas. Porém essas variantes conservam como base uma cruz quadrada e escalonada, conforme são apresentadas na Figura 2.

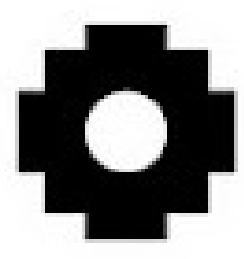

(a)

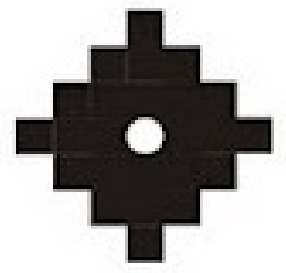

(b)

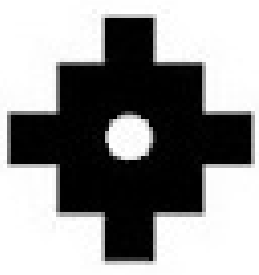

(c)

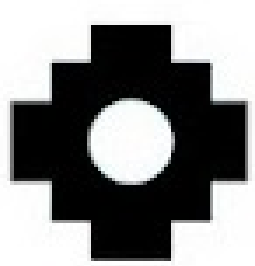

(d)

Figura 2: Algumas variações estilísticas do símbolo da chakana

Fonte: 
Para o trabalho neste artigo utilizaremos figura geométrica da chakana ilustrada na Figura 2a.

De acordo com Bazán, Villena, Ramos e Rengifo [2, 8, 10, 11], algumas das culturas pré-incas que usaram a chakana são: Caral, Chavin, Chincay, Mochica, Paracas, Chiribaya e Tiauanaco, sendo a cultura inca ou Tahuantinsuyo (tahua significa quatro) a última que usou este elemento simbólico. Os 4 lados maiores correspondem à divisão do império inca em 4 suyos (estados): Chinchaysuyo, Antisuyo, Collasuyo e Contisuyo. O círculo central representa Cuzco, o umbigo do mundo.

Segundo Lajo [4], a Qhapaq Nan, é consistente com a geometria da chakana. É uma estrada em linha reta que passa por várias cidades do império inca como Cajamarca, Cuzco, Tiahuanaco, Oruo e Potosí, como podemos observar na Figura 3.
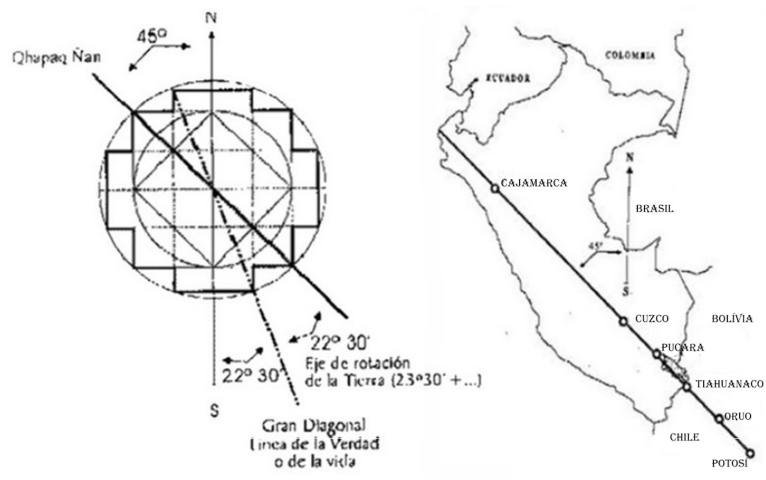

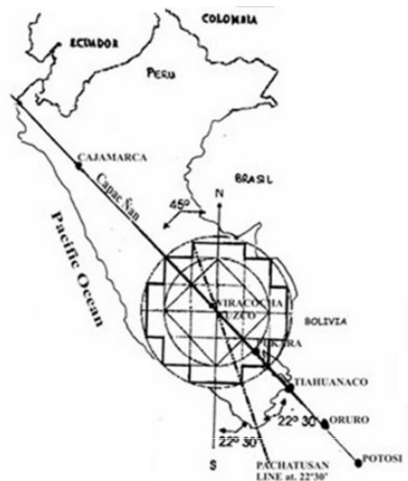

Figura 3: Trajetória descrita pela Qhapaq Ñan

Fonte: https://etoiles.noblogs.org/post/2015/07/07/wiphala/

No trabalho de Bazán [2] são exibidas diversas figuras de vestígios arqueológicos de algumas culturas pré-incaicas onde aparece a chakana. Como exemplo, a Figura 4 mostra parte do obelisco Tello da cultura pré-incaica Chavín (1200 a.C - 400 a.C.) desenvolvida no norte de Peru. 


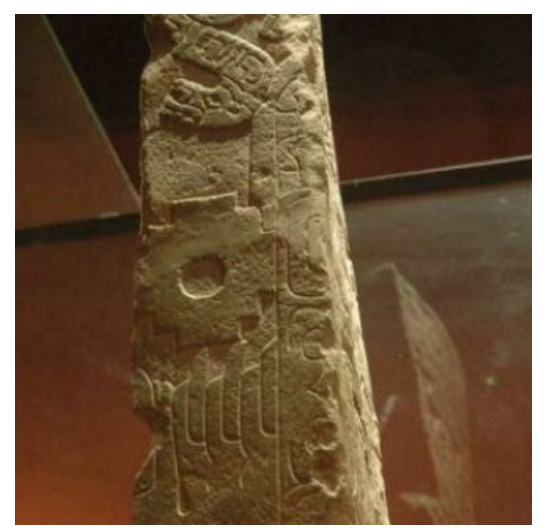

Figura 4: A chakana esculpida no Obelisco Tello

Fonte: Extraído de Bazán [2] p. 220

Segundo Lambert [5], a chakana ou chaka hanan significa ponte ao alto/escada, representa diversos conceitos e princípios, segundo seu uso. De acordo com Villena [8], símbolo com importantes funções para os andinos em vários aspectos: astronômico, agrícola, espiritual, arquitetônico e matemático. Cada um dos seus degraus representa os meses do ano, a parte superior, inferior e laterais representam as quatros estações do ano, servindo como um calendário agrícola para as épocas de plantio e colheita, conforme mostra a Figura 5.

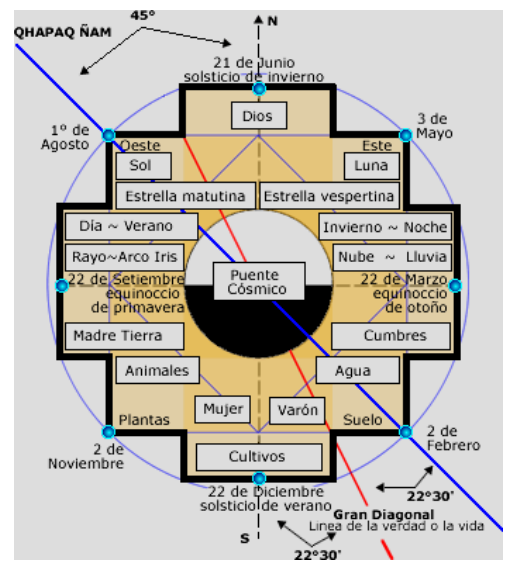

Figura 5: Utilidades da chakana

Fonte: 
Com a figura geométrica da chakana podemos demonstrar o Teorema de Pitágoras e estudar conceitos geométricos importantes como circunferência, diâmetro, raio, quadrados inscritos e circunscritos, diagonais, ponto médio, área e perímetro.

\subsection{Construção geométrica da chakana}

Para dar início à construção geométrica da chakana, primeiramente vamos apresentar uma expansão de quadrados utilizando as diagonais e circunferências.

Na Figura 6 é apresentada uma sequência de desenhos geométricos em que cada quadrado tem o lado com mesma medida da diagonal do quadrado anterior.

Considere:

1. um quadrado inicial $\mathrm{A}_{1} \mathrm{~B}_{1} \mathrm{C}_{1} \mathrm{D}_{1}$ de lado unitário e uma circunferência $\mathscr{C}_{1}$ de centro $\mathrm{O}$, inscrita neste quadrado de diâmetro igual a uma unidade;

2. uma circunferência $\mathscr{C}_{2}$ com diâmetro de comprimento $\sqrt{2}$ circunscrita ao quadrado $\mathrm{A}_{1} \mathrm{~B}_{1} \mathrm{C}_{1} \mathrm{D}_{1}$;

3. o quadrado $\mathrm{A}_{2} \mathrm{~B}_{2} \mathrm{C}_{2} \mathrm{D}_{2}$ de lado medindo $\sqrt{2}$, circunscrito a $\mathscr{C}_{2}$ de modo que os vértices do quadrado $\mathrm{A}_{1} \mathrm{~B}_{1} \mathrm{C}_{1} \mathrm{D}_{1}$ são os pontos médios do quadrado de seus lados;

4. uma circunferência $\mathscr{C}_{3}$ com diâmetro de comprimento 2 circunscrita ao quadrado $\mathrm{A}_{2} \mathrm{~B}_{2} \mathrm{C}_{2} \mathrm{D}_{2}$;

5. um quadrado $\mathrm{A}_{3} \mathrm{~B}_{3} \mathrm{C}_{3} \mathrm{D}_{3}$ de lado medindo 2, circunscrito a $\mathscr{C}_{3}$ de modo que os vértices do quadrado $\mathrm{A}_{2} \mathrm{~B}_{2} \mathrm{C}_{2} \mathrm{D}_{2}$ são os pontos médios de seus lados;

6. uma circunferência $\mathscr{C}_{4}$ com diâmetro de comprimento $2 \sqrt{2}$ circunscrita ao quadrado $\mathrm{A}_{3} \mathrm{~B}_{3} \mathrm{C}_{3} \mathrm{D}_{3}$;

7. um quadrado $\mathrm{A}_{4} \mathrm{~B}_{4} \mathrm{C}_{4} \mathrm{D}_{4}$ de lado medindo $2 \sqrt{2}$ circunscrito a $\mathscr{C}_{3}$ de modo que os vértices do quadrado $\mathrm{A}_{3} \mathrm{~B}_{3} \mathrm{C}_{3} \mathrm{D}_{3}$ são os pontos médios de seus lados;

8. finalmente, uma circunferência $\mathscr{C}_{5}$ com diâmetro de comprimento 4 circunscrita ao quadrado $\mathrm{A}_{4} \mathrm{~B}_{4} \mathrm{C}_{4} \mathrm{D}_{4}$. 


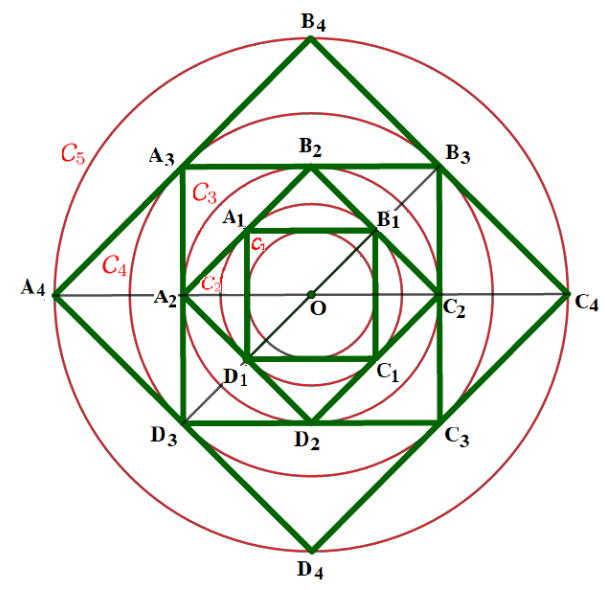

Figura 6: Expansão de quadrados

Vamos utilizar o princípio da expansão de quadrados apresentado na Figura 6 para dar início à construção da Chakana:

- Vamos interromper a expansão dos quadrados no quadrado $\mathrm{A}_{3} \mathrm{~B}_{3} \mathrm{C}_{3} \mathrm{D}_{3}$ e na circunferência $\mathscr{C}_{4}$ que circunscreve este quadrado (Figura 7).

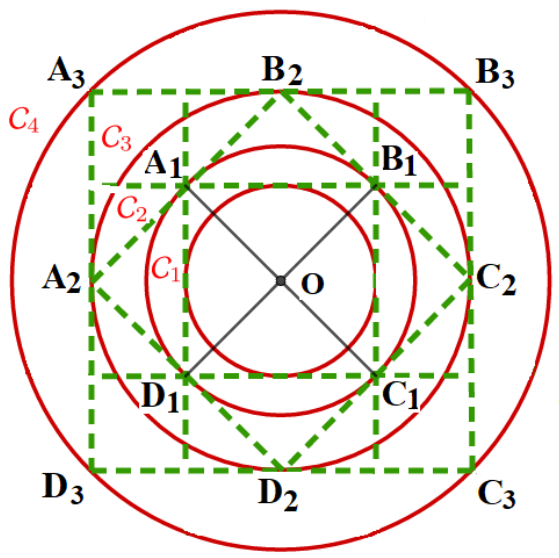

Figura 7: Quadrado $\mathrm{A}_{3} \mathrm{~B}_{3} \mathrm{C}_{3} \mathrm{D}_{3}$ inscrito na circunferência $\mathscr{C}_{4}$

- Prolongando os lados do quadrado $A_{1} B_{1} C_{1} D_{1}$, obtemos os pontos $E_{1}, F_{1}, G_{1}, H_{1}, I_{1}, J_{1}, K_{1}, L_{1}$, interseções dos prolongamentos com $\mathscr{C}_{4}$ (Figura 8). 


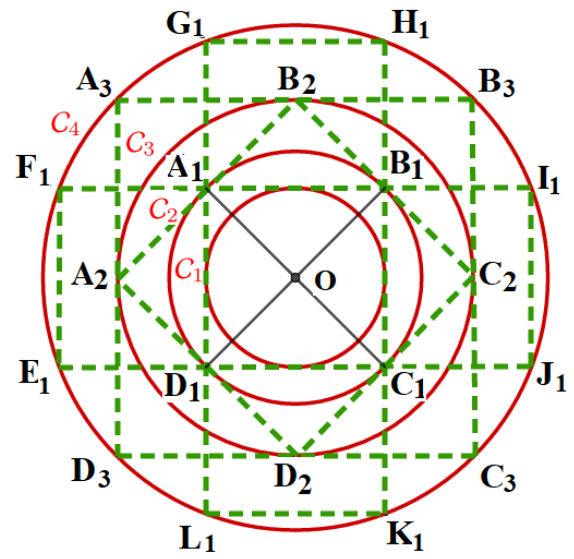

Figura 8: Prolongamento dos lados do quadrado $\mathrm{A}_{1} \mathrm{~B}_{1} \mathrm{C}_{1} \mathrm{D}_{1}$

- Após o prolongamentos dos lados do quadrado $\mathrm{A}_{1} \mathrm{~B}_{1} \mathrm{C}_{1} \mathrm{D}_{1}$, obtemos a forma geométrica ilustrada na Figura 9.

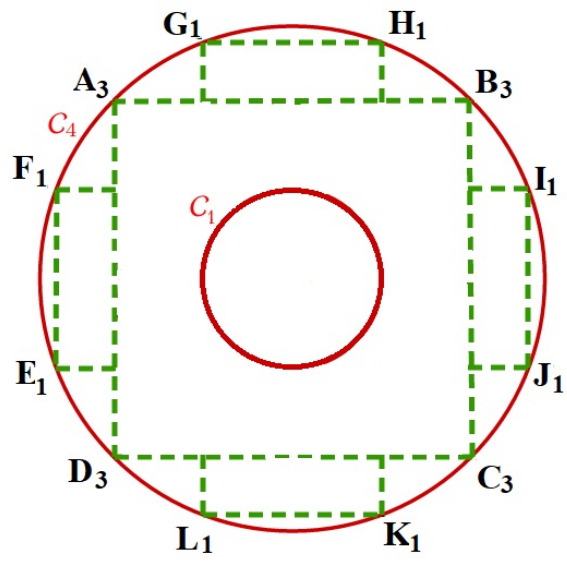

Figura 9: Figura geométrica formada

- A chakana é o símbolo geométrico inscrito na circunferência $\mathscr{C}_{4}$ com um buraco no centro representado pela circunferência $\mathscr{C}_{1}$ (Figura 10). 


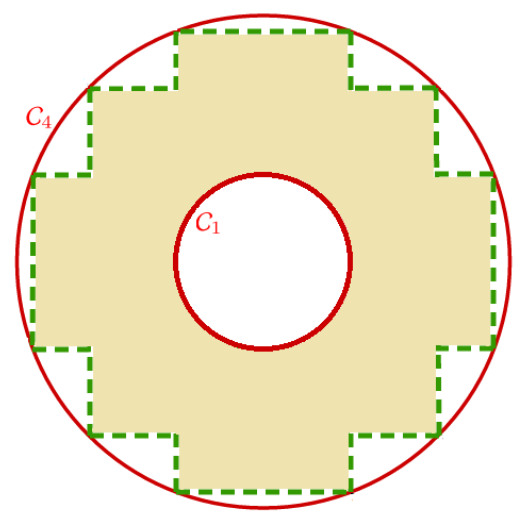

Figura 10: A Chakana

\subsection{Explorando o teorema de Pitágoras na chakana}

Utilizando da mesma forma geométrica daChakana construída na Figura 9 e não levando em consideração o pequeno círculo central, vamos explorar o teorema de Pitágoras, enunciado a seguir.

Teorema 1. Em todo triângulo retângulo, o quadrado do comprimento da hipotenusa é igual à soma dos quadrados dos comprimentos dos catetos, isto é, dado um triângulo retângulo de catetos com comprimentos b e c e hipotenusa a, vale a relação:

$$
\mathrm{a}^{2}=\mathrm{b}^{2}+\mathrm{c}^{2}
$$

Demonstração. Considere o quadrado $\mathrm{A}_{3} \mathrm{~B}_{3} \mathrm{C}_{3} \mathrm{D}_{3}$ da Figura 9 dividido em pequenos quadrados. No interior do quadrado $\mathrm{A}_{3} \mathrm{~B}_{3} \mathrm{C}_{3} \mathrm{D}_{3}$ construímos o quadrado $\mathrm{ABCD}$ com lados de comprimento a e $A_{3} A=B_{3} B=C_{3} C=D_{3} D=\mathbf{c}, D_{3}=A_{3}=B_{3}=C_{3}=\mathbf{b}$, conforme Figura 11 .

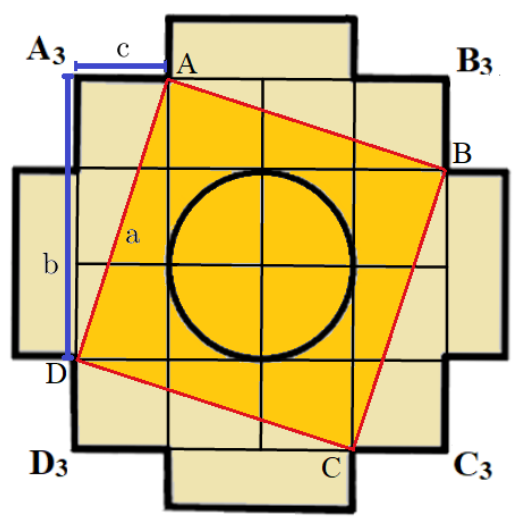

Figura 11: Teorema de Pitágoras na Chakana 
Dessa forma, pode-se afirmar que os lados do quadrado $\mathrm{A}_{3} \mathrm{~B}_{3} \mathrm{C}_{3} \mathrm{D}_{3}$ têm comprimento $(\mathbf{c}+\mathbf{b})$ e que sua área será igual à soma das áreas dos quatro triângulos retângulos congruentes de catetos de comprimentos $\mathbf{c}$ e $\mathbf{b}$ e hipotenusa de comprimento a, com a área do quadrado ABCD.

Portanto, temos que

$$
4 \frac{\mathbf{b c}}{2}+\mathbf{a}^{2}=(\mathbf{b}+\mathbf{c})^{2} \Longrightarrow 2 \mathbf{b c}+\mathbf{a}^{2}=\mathbf{b}^{2}+2 \mathbf{b c}+\mathbf{c}^{2}
$$

Logo,

$$
\mathbf{a}^{2}=\mathbf{b}^{2}+\mathbf{c}^{2}
$$

Assim fica demonstrado o teorema de Pitágoras.

\section{3. Área da chakana e comprimento de sua fronteira}

Utilizaremos a chakana construída na subseção 3.1 para determinarmos sua área e o comprimento do sua fronteira.

Observe que podemos dividir a chakana em quatro retângulos congruentes, sendo um deles o retângulo $\mathrm{G}_{3} \mathrm{G}_{1} \mathrm{H}_{1} \mathrm{H}_{3}$ e o quadrado $\mathrm{A}_{3} \mathrm{~B}_{3} \mathrm{C}_{3} \mathrm{D}_{3}$ e na circunferência $\mathscr{C}_{1}$ de centro no ponto $\mathrm{O}$, conforme mostra a Figura 12.

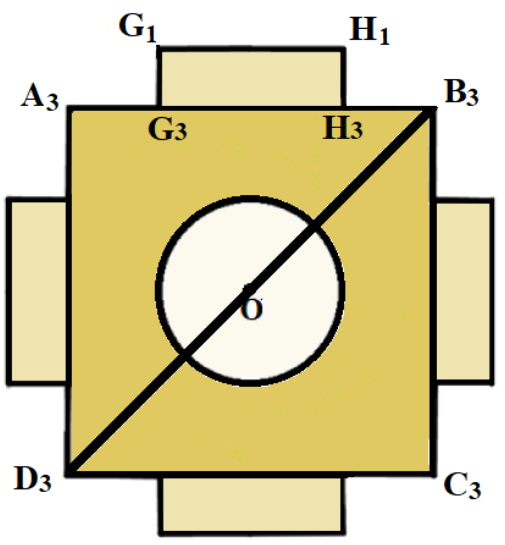

Figura 12: Quadrado $\mathrm{A}_{3} \mathrm{~B}_{3} \mathrm{C}_{3} \mathrm{D}_{3}$ e quatro retângulos congruentes

Denotemos por $L$ o comprimento do lado do quadrado $A_{3} B_{3} C_{3} D_{3}$, então suas diagonais terão comprimento $L \sqrt{2}$

$\mathrm{Na}$ Figura 13, observe que o quadrado $\mathrm{A}_{3} \mathrm{~B}_{3} \mathrm{C}_{3} \mathrm{D}_{3}$ pode ser dividido em 16 outros quadrados menores, assim o que o raio da circunferência $\mathscr{C}_{1}$ tem comprimento $\frac{\mathrm{L}}{4}$, o raio da circunferência 
$\mathscr{C}_{4}$, circunscrita ao quadrado $\mathrm{A}_{3} \mathrm{~B}_{3} \mathrm{C}_{3} \mathrm{D}_{3}$ mede $\frac{\mathrm{L} \sqrt{2}}{2}$ unidades, portanto no triângulo $\Delta \mathrm{OPH}_{1}$, reto em $\mathrm{P}$, temos que $\overline{\mathrm{OP}}=\frac{\mathrm{L}}{4}$ e $\overline{\mathrm{OH}_{1}}=\frac{\mathrm{L} \sqrt{2}}{2}$.

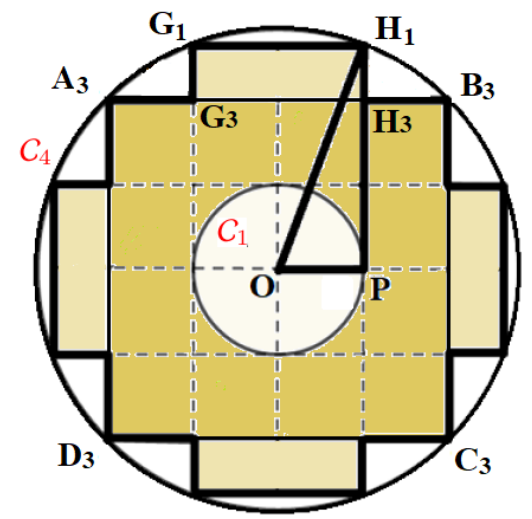

Figura 13: Comprimentos dos raios de $\mathscr{C}_{1}$ e de $\mathscr{C}_{4}$

Vamos determinar o comprimento do segmento $\overline{\mathrm{H}_{1} \mathrm{P}}$ pelo teorema de Pitágoras. Tem-se

$$
\left(\frac{\mathrm{L} \sqrt{2}}{2}\right)^{2}=\left(\frac{\mathrm{L}}{4}\right)^{2}+{\overline{\mathrm{H}}{ }_{1} \mathrm{P}^{2}}_{\Rightarrow}{\overline{\mathrm{H}_{1}}}^{2}=\frac{2 \mathrm{~L}^{2}}{4}-\frac{\mathrm{L}^{2}}{16}=\frac{7 \mathrm{~L}^{2}}{16} \Rightarrow \overline{\mathrm{H}_{1} \mathrm{P}}=\frac{\mathrm{L} \sqrt{7}}{4}
$$

Como $\overline{\mathrm{H}_{3} \mathrm{P}}=\frac{\mathrm{L}}{2}$, segue que $\overline{\mathrm{H}_{1} \mathrm{H}_{3}}=\frac{\mathrm{L} \sqrt{7}}{4}-\frac{\mathrm{L}}{2}=\frac{\mathrm{L}(\sqrt{7}-2)}{4}$.

Portanto,

$$
\overline{\mathrm{H}_{1} \mathrm{H}_{3}}=\frac{L(\sqrt{7}-2)}{4} .
$$

Proposição 1. A fronteira da chakana, $\mathrm{F}_{\mathrm{chak}}$, é dada por

$$
\mathrm{F}_{\text {chak }}=\frac{\mathrm{L}}{2}(4 \sqrt{7}+\pi) \text { u.m. }
$$

Demonstração. A fronteira da chakana é composta por: 8 segmentos congruentes a $\overline{\mathrm{H}_{3} \mathrm{~B}_{3}}$, de comprimento $\frac{\mathrm{L}}{4}, 8$ segmentos congruentes a $\overline{\mathrm{H}_{3} \mathrm{H}_{1}}$, cujo comprimento é dado na Equação (1), 4 segmentos congruentes a $\overline{\mathrm{G}_{1} \mathrm{H}_{1}}$, de comprimento $\frac{\mathrm{L}}{2}$ e o perímetro de uma circunferência central $\mathscr{C}_{1}$ de raio $\frac{L}{4}$, desse modo temos: 


$$
\mathrm{F}_{\text {chak }}=8\left(\frac{\mathrm{L}}{4}\right)+8 \frac{\mathrm{L}(\sqrt{7}-2)}{4}+2 \pi\left(\frac{\mathrm{L}}{4}\right)+4\left(\frac{\mathrm{L}}{2}\right) \text { u.m. }
$$

Reduzindo os termos algébricos do Equação (2), a fronteira da chakana é dada por:

$$
\mathrm{F}_{\text {chak }}=\frac{\mathrm{L}}{2}(4 \sqrt{7}+\pi) \text { u.m. }
$$

Proposição 2. A área da região definida pela chakana, $\mathrm{A}_{\mathrm{chak}}$, é dada por

$$
\mathrm{A}_{\text {chak }}=\frac{\mathrm{L}^{2}}{16}(8 \sqrt{7}-\pi) \text { u.a. }
$$

Demonstração. A área da chakana é dada pela adição da área do quadrado $\mathrm{A}_{3} \mathrm{~B}_{3} \mathrm{C}_{3} \mathrm{D}_{3}$ com as áreas dos quatro retângulos congruentes, subtraindo a área do pequeno círculo central de raio $\frac{\mathrm{L}}{4}$. Denotemos por $A_{1}$ a área do quadrado $A_{3} B_{3} C_{3} D_{3}$, assim,

$$
\mathrm{A}_{1}=\left(\overline{\mathrm{A}_{3} \mathrm{~B}_{3}}\right)\left(\overline{\mathrm{B}_{3} \mathrm{C}_{3}}\right)=\mathrm{L}^{2} \text { u.a. }
$$

Denotemos por $A_{2}$ as áreas dos retângulos, logo

$$
A_{2}=4\left(\overline{\mathrm{G}_{1} \mathrm{H}_{1}}\right)\left(\overline{\left.\mathrm{H}_{1} \mathrm{H}_{3}\right)}=4\left(\frac{\mathrm{L}}{2}\right)\left(\frac{\mathrm{L}}{4}\right)(\sqrt{7}-2)=\frac{\mathrm{L}^{2}}{2}(\sqrt{7}-2)\right. \text { u.a. }
$$

Por último, denotemos por $A_{3}$ a área do pequeno círculo central de raio $\frac{\mathrm{L}}{4}$, $\operatorname{logo}$

$$
\mathrm{A}_{3}=\frac{\pi \mathrm{L}^{2}}{16} \text { u.a. }
$$

Assim, das Equações (3), (4) e (5), temos:

$$
A_{\text {chak }}=L^{2}+\frac{L^{2}}{2}(\sqrt{7}-2)-\frac{\pi L^{2}}{16}=L^{2}+\frac{L^{2} \sqrt{7}-2 L^{2}}{2}-\frac{\pi L^{2}}{16} \text { u.a. }
$$

Simplificando os termos da Equação (6), a área da chakana é dada por

$$
\mathrm{A}_{\text {chak }}=\frac{\mathrm{L}^{2}}{16}(8 \sqrt{7}-\pi) \text { u.a. }
$$




\section{Interdisciplinaridade}

A história das civilizações antigas, utilizada no ambiente escolar pode estimular a pesquisa e permitir que várias disciplinas trabalhem em conjunto.

De acordo com Bazán [2] há uma possível ligação histórico-cultural entre os povos andinos précolombianos e a cultura Kadiwéu do Brasil por meio da simbologia da chakana nas diversas expressões artísticas.

É previsto na BNCC [3] as habilidades: "Identificar os espaços territoriais ocupados e os aportes culturais, científicos, sociais e econômicos dos astecas, maias e incas e dos povos indígenas de diversas regiões brasileiras"e "Identificar algumas constelações no céu, com o apoio de recursos (como mapas celestes e aplicativos digitais, entre outros), e os períodos do ano em que elas são visíveis no início da noite".

Nesta seção apresentamos sugestões de algumas disciplinas que podem ser envolvidas nesse contexto.

\subsection{História}

O professor de História pode solicitar pesquisas sobre o uso da chakana e a agricultura, e a relação entre os povos andinos e o povo Kadiwéu ${ }^{2}$. Algumas dessas relações manifestam-se no símbolo da chakana, como ilustrado na Figura 14.

\footnotetext{
${ }^{2}$ Povo indígena brasileiro. Lutaram pelo Brasil na Guerra do Paraguai.
} 


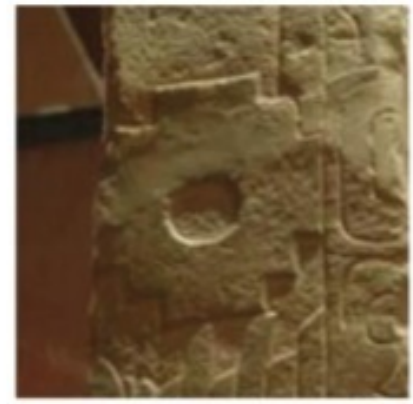

(a) Obelisco Telles da cultura Chavin (Peru) (1220 a.C - 200 a.C)

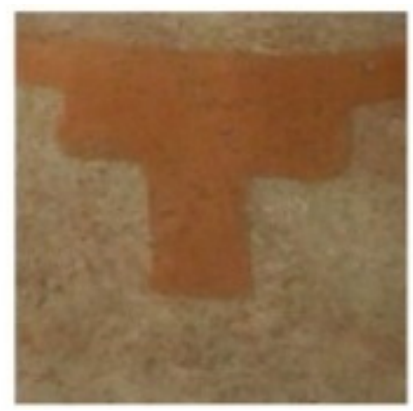

(c) Vaso cerimonial: Arte na cultura Moche (Peru) (200 a.C - 500 d.C)

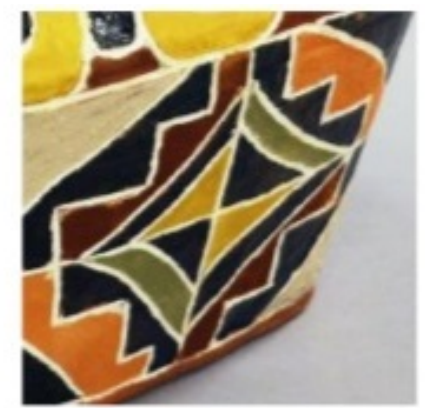

(b) Arte Kadiwéu (Brasil) (Séc. XVII)

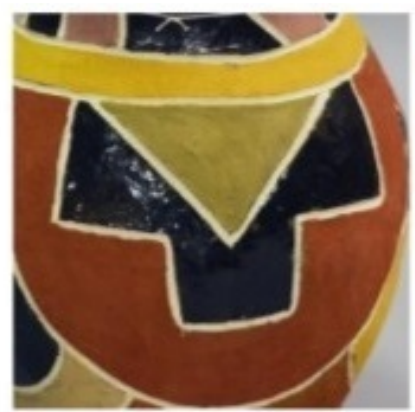

(d) Arte Kadiwéu (Brasil) (Sec. XVII)

Figura 14: Semelhança entre a cultura andina e a Kadiwéu

Fonte: Extraído de Bazán [2] p. 180

\subsection{Artes}

O professor de Artes pode explorar o artesanato, pinturas em cerâmicas. Na Figura 15 são ilustradas algumas formas de arte em que a chakana está presente, como arte têxtil de Nazca (300 a.C - 800 d.C) com cruzes quadradas, Figura 15a; a cerâmica da cultura La Tolita com representação da cruz quadrada, Figura 15b; a imagem de um vaso em cerâmica em forma de cabeça com uma chakana desenhada na testa, Figura 15c; e a arte Kadiwéu ilustrada nas figuras 14b e 14d. 


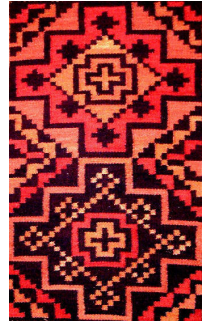

(a)

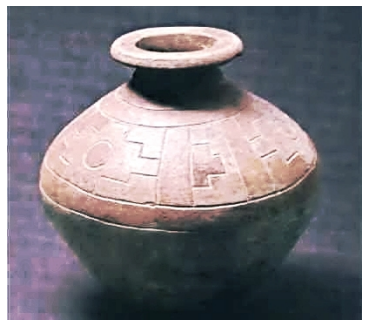

(b)

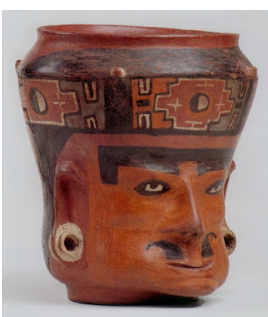

(c)

Figura 15: Chakana na arte da cultura pre-incaica Nazca

Fonte: https://peregrinadanza.wordpress.com/2015/10/10/las-cruces-cuadradas-chavin/

\subsection{Geografia}

O professor de geografia pode abordar a civilização inca na América do Sul e explorar a localização, extensão, exibida na Figura 16a, e o caminho Qhapaq Ñan [4], Figura 3, o relevo dos Andes, falhas geológicas, e resistência das construções arquitetônicas. Na Figura 16b é vista uma parte do sítio arqueológico de Machu Picchu, uma cidade inca localizada a 2400 metros de altitude, no Peru.

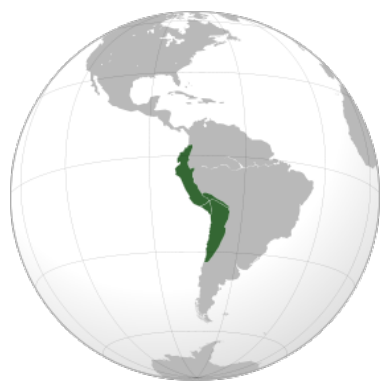

(a)

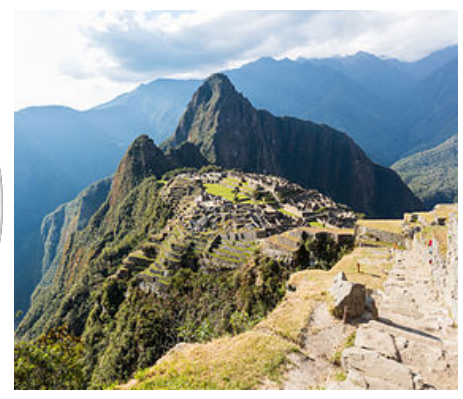

(b)

Figura 16: Mapa e relevo

Fonte: https://pt.wikipedia.org/wiki/Imp\%C3\%A9rio_Inca

\subsection{Ciências com astronomia}

O professor de Ciências pode solicitar que os alunos observem o céu noturno e estrelado e identifiquem algumas estrelas como as do Cinturão de Orion (Três Marias), visualizadas na Figura 17a, e as estrelas da constelação Cruzeiro do Sul, visualizadas na Figura 17b, e relacionar essa constelação com a chakana que era usada como orientação espacial dos Incas. 


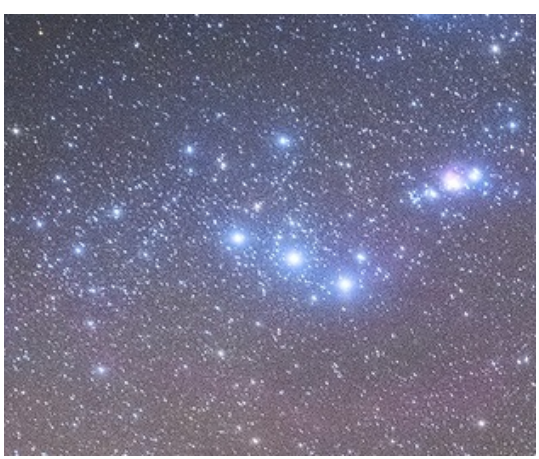

(a) Cinturão de Orion

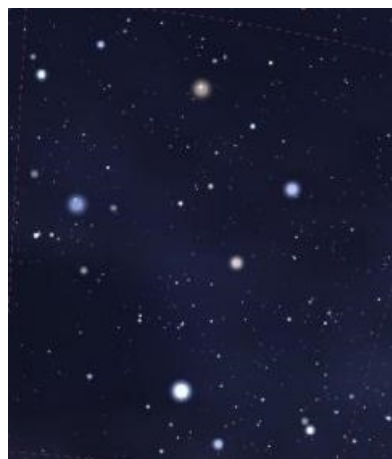

(b) Cruzeiro do Sul

Figura 17: Constelações

Fonte: http://www.astropt.org/

\section{Conclusão}

A História da Matemática pode ser utilizada como elemento motivador em particular para o estudo da geometria, mostrando aos alunos como ela se desenvolveu em diferentes culturas de civilizações antigas.

A geometria e sua conexão com a história das antigas civilizações é uma prática metodológica interdisciplinar, a qual pode nos fornecer uma variedade de situações conextualizadas a serem exploradas em sala de aula, tais como: seus costumes, construções arquitetônicas e pinturas artísticas, além de favorecer à alfabetização cartográfica e com isso estimular a interdisciplinaridade.

Os conhecimentos históricos, a importância da geometria e curiosidades presentes nesses assuntos são elementos que podem despertar no aluno um senso mais crítico e uma maior capacidade para pesquisar sobre as raizes de seus antepassados, assim como também buscar soluções para diferentes situações encontradas no cotidiano.

\section{Referências}

[1] Andrade, L. C. Aspectos Geométricos de Alguns Símbolos Místicos. Dissertação de Mestrado (Profmat). 2018. Disponível em: <https://sca.profmat-sbm.org.br/sca_v2/get_tcc3.php?id= 161030208>. Acesso em: 05 de janeiro de 2021.

[2] Bazán, P. S. Evidências e reflexões sobre uma possível ligação histórico-cultural entre os povos andinos pré-colombianos e a cultura kadiwéu do Brasil por meio da simbologia Chakana nas diversas expressões artísticas. Dissertação (Mestrado em Artes). Universidade Estadual Paulista "Júlio de Mesquita Filho", São Paulo, 2020.

[3] BNCC. Base Nacional Comum Curricular. Disponível em: <http://basenacionalcomum.mec. gov.br/images/BNCC_EI_EF_110518_versaofinal_site.pdf $>$. Acesso em: 20 de dezembro de 2020 . 
[4] Lajo, J. Qhapaq ñan: a rota inca da sabedoria. 2006. Disponível em: <https://digitalrepository. unm.edu/abya_yala/358>. Acesso em: 27 de janeiro de 2021.

[5] Lambert, M. Chakana, a cruz andina. 2016. Disponível em: <http://blog.marcelolambert.com/ search?q=chakana $>$. Acesso em: 20 de dezembro de 2020.

[6] Maldonado, A. P. Significado dos Símbolos: Cruz Inca ou Chakana. 2016. Disponível em: <https://www.altoastral.com.br/significado-dos-simbolos-cruz-inca-ou-chakana/>. Acesso em: 20 de dezembro de 2020.

[7] Motta, S. A pedra de 12 ângulos feita pelos incas em Cusco. Disponível em: <http:// sergiomotta.com.br/a-pedra-de-12-angulos-feita-pelos-incas-em-cusco/>. Acesso em: 03 de fevereiro de 2021.

[8] Villena, C. M. Genesis de la Cultura Andina. Fondo Editorial C.A.P.. Lima, Peru, 1983.

[9] Amaral, M. M. DO, Márquez, R. M. G., Araújo, J. C. DE. "Algumas velocidades de órbitas planetárias". REMAT: Revista Eletrônica da Matemática, v. 6, nº1, p. 1-14, 30 dez. 2019.

[10] Ramos, Jorge R. Análisis comparativo entre el diseño iconográfico andino precolombino y actual del Ecuador com el Peru y Bolivia. 220 f. Monografia (Bacharel em Design Gráfico) Faculdade de Ciência da Computação e Eletrônica, Escola de Design Gráfico, Escola Superior Politécnica Chimborazo, Equador, 2009.

[11] Rengifo, M. G. F. La Chakana y los saberes ancestrales del Pueblo Kayambi. Trabalho de Conclusão de Curso (Licenciatura em ciências da educação). Universidad central del Ecuador. 2018

[12] Servindi, Mundo andino celebra o Dia da Chakana. Disponível em: <https://www.servindi. org/actualidad-noticias/03/05/2019/el-dia-de-la-chakana>. Acesso em: 03 de fevereiro de 2021.

Luiz Carlos de Andrade ETE Helber Vignoli Muniz - FAETEC

<luizsaqu@gmail.com>

Rosa García Márquez Faculdade de Formação de Professores - UERJ <rgmalillo@gmail.com>

Fábio Silva de Souza

Faculdade de Formação de Professores - UERJ $<$ fasouza08@gmail.com>

Recebido: 05/02/2021

Publicado: 01/06/2021 